\title{
Characterization of a circular thin film plasma source for atomic emis- sion spectroscopy
}

\author{
J. M. SWAN* and R. D. SACKs ${ }^{\dagger}$ \\ Department of Chemistry, University of Michigan, Ann Arbor, MI 48109, U.S.A.
}

(Received 27 February 1985; in revised form 21 May 1985)

\begin{abstract}
A plasma source for analytical atomic emission spectroscopy is described based on the electrical vaporization by capacitive discharge of a thin $\mathrm{Ag}$ film deposited on a polycarbonate membrane filter. The source is designed for the rapid, direct analysis of solid powder samples collected by filtration from fluid media. A concentric electrode system consisting of a ring-shaped graphite electrode placed on the thin film surface and a pointed graphite rod located under the membrane substrate results in a plasma with cylindrical symmetry and a radial current path. Discharge current vs time and intensity vs time profiles are compared for the concentric electrode geometry and the linear geometry used in previous studies. Two values of tank circuit inductance also are compared. Both neutralatom and ion line radiation from an $\mathrm{Mn}$ sample are more intense when the center electrode is initially cathodic. The inside diameter of the ring-shaped electrode and thus the surface area of the $\mathrm{Ag}$ film exposed to the plasma have relatively little effect on the intensity of continuum background and line radiation from a sample deposited near the center of the film. Particle size effects, while significant, are smaller than with the linear electrode geometry. Analytical curves are presented for several lines using both a low-inductance and a high-inductance discharge. Log-log slopes range from about 0.85 to 1.05 for ion lines. Detection limits are somewhat poorer than with the linear geometry.
\end{abstract}

\section{INTRODUCTION}

THE HIGH-TEMPERATURE plasmas produced by the electrical vaporization of thin metal films deposited on polymer substrates have been used for the direct determination by emission spectroscopy of metallic elements in a variety of solid, powder samples [1,2]. Concomitant effects are minimal, and in most cases, a single analytical curve for the element of interest can be used for the determination of that element over a wide range of sample composition. Any reagent or other material containing the analyte element can be used as the analytical standard. Relative errors in the 5-15\% range are routinely obtained without any attempt at matching the overall composition of the standards and samples.

The effect of sample particle size on radiation intensity is significant if the intensity is integrated from the start of the discharge. However, particle size effects are minimal for sample particles as large as about $30 \mu \mathrm{m}$ if a delayed integration interval is used in conjunction with a high-inductance dischargc. Delayed integration with an $8-\mathrm{kV}, 30-\mu \mathrm{F}$, $1200-\mu \mathrm{H}$ discharge also permits the analysis of samples as large as $1.0 \mathrm{mg}$ with no significant sample loading effects. This results in relative detection limits in the low and sub part-permillion range for many metallic elements.

A number of important solid samples occur as suspensions in fluid media. Examples include wear metals in lubricating oil, coal slurries, airborne particulate material, and solids suspended in natural waters and industrial effluents. CLARK and SACKS [3] showed that the emission spectra of metallic elements in refractory powder samples could be obtained by filtering the samples through $47-\mathrm{mm}$ diameter polycarbonate membrane filters which were previously coated with a thin silver film. They showed that a bright, highly reflective metallic film could be obtained which did not affect the filtration properties of the membranes.

\footnotetext{
* Present address: Department of Chemistry, University of Wisconsin-River Falls, River Falls, WI 54022, U.S.A.

† Author to whom correspondence should be directed.
} 
Graphite electrodes were placed across a diameter of the circular metal film, and a 5-kV, 281-J discharge was used to vaporize the film and generate a plasma in the metal vapor.

Sur [4] developed a concentric electrode arrangement for these circular metal films on membrane filters. A ring-shaped graphite outer electrode is placed on the film surface, and a pointed graphite pin is placed in contact with the underside of the $0.01-\mathrm{mm}$ thick polycarbonate membrane. While there is no electrical contact between the electrodes, dielectric breakdown occurs through the filter pores as soon as the capacitor voltage is switched across the electrodes. Unlike the plasmas produced with the previously used linear electrode geometry, the plasmas from the concentric electrode system have a symmetry axis, and spatially-resolved radiation measurement can be deconvoluted to obtain plasma emissivity values. SUH and SACKS [5] used this feature to measure the time and spatiallyresolved electronic excitation temperature, electron number density and the degree of ionization of an added probe element.

SWAN and SACKs [6] recently used this system for the direct determination of $\mathrm{Pb}$ in urban airborne particulate material (U.S. National Bureau of Standards reference material SRM1648 ) and in lubricating oil spiked with $\mathrm{Pb}$ powder. Alcohol suspensions of the urban particulate material and petroleum ether dilutions of the oil were pulled through the metallized membrane filter and the powder residues vaporized and excited in the electrically vaporized thin film plasmas. Sample recoveries ranged from 74 to $109 \%$ using alcohol suspensions of $\mathrm{Pb}$ powder as calibration standards.

The study reported here provides a more detailed analytical characterization of the circular thin film plasma source and a comparison of it with the linear thin film plasma source used routinely in this laboratory for the direct determination of metallic elements in solid, powder samples.

\section{Apparatus and Techniques}

\subsection{Capacitive discharge circuit and associated apparatus}

The essential features of the capacitive discharge circuit are shown in Fig. 1. Component values are listed in Table 1. This circuit is a modified version of the discharge circuits used in previous electrically vaporized thin film studies $[7,8]$. The circuit contains a $12-\mathrm{kV}, 60-\mathrm{mA}$, center-tap transformer the output of which is rectified by two diode stacks. The current limiting resistor $R 1$ leads to the main discharge capacitor $\mathrm{C}$ which can be adjusted from $7.5 \mu \mathrm{F}$ to $30 \mu \mathrm{F}$ in $7.5-\mu \mathrm{F}$ steps. Divider network $\mathrm{R} 3$ and R4 is used to monitor the capacitor voltage by digital voltmeter V. Switches S1, S2 and S3 are all gravity operated spark-gap relays as described by SuH et al. [8]. Switch S1 is the main discharge control switch used to initiate the discharge on command. Switch S2 together with resistor R2 is used to discharge residual capacitor voltage following each experiment. The discharge current is monitored by a broad-band current-to-voltage transformer (I). The inductor $\mathrm{L}$ is either the $5 \mu \mathrm{H}$ residual circuit inductance for a short duration discharge or a $1200-\mu \mathrm{H}$ added inductance for a longer duration discharge.

With previous discharge circuits used for electrically vaporized thin film studies, the discharge rapacitor was charged directly through the thin metal film. With the concentric system, that is not possible since the two electrodes are separated by the dielectric polycarbonate membrane and thus represent an open circuit prior to dielectric breakdown during the discharge. Switch $\$ 3$ together with the resistor R6 is used to bypass the thin film during capacitor charging. Switch S3 is opened prior to initiating the discharge. When $\mathbf{S} 3$ is open, the capacitor has no ground reference and a significant voltage may develop across the two electrodes in the film holder. Often a minor discharge occurs burning the membrane before the main discharge is initiated. In addition, the capacitor voltage cannot be monitored by the resistive divider. Both problems are eliminated by including resistor R5 in the circuit.

All experiments were conducted in a nylon discharge chamber which is similar to previous designs [1, 3]. The chamber is fitted with a quartz observation window and gas flow lines. All experiments were

[4] S. Y. SuH, Ph.D. Dissertation, University of Michigan, Ann Arbor, MI (1981).

[5] S. Y. SUH and R. D. SACKS, Spectrochim. Acta 36B, 1081 (1981).

[6] J. M. SWAN and R. D. SACKS, Anal. Chem., in press (1985).

[7] D. V. Duchane and R. D. SACKs, Anal. Chem. 50, 1752 (1978).

[8] S. Y. SUH, R. J. Collins and R. D. SACKs, Appl. Spectrosc. 35, 42 (1981). 


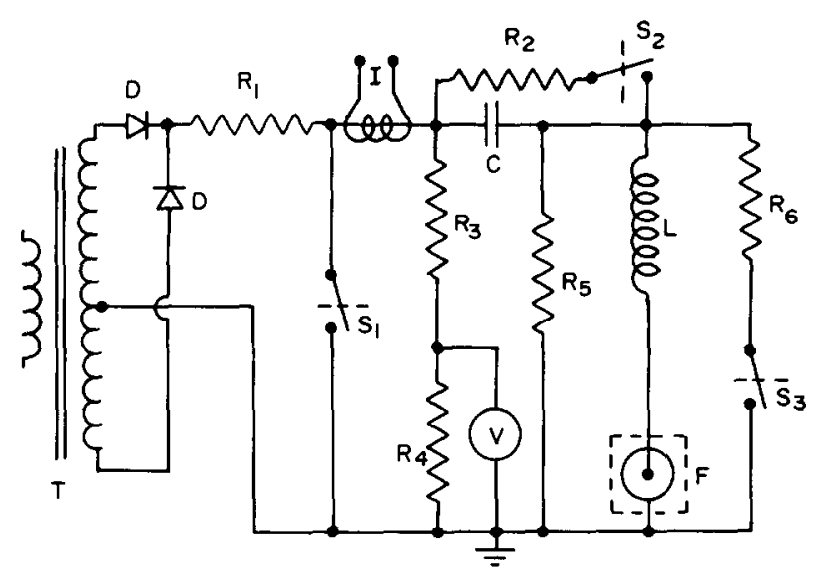

Fig. 1. Simplified schematic of high-voltage discharge circuit. Component values are given in Table 1.

Table 1. Main discharge circuit components

\begin{tabular}{ll}
\hline $\mathbf{R}_{1,5}$ & $100 \mathrm{k} \Omega, 100 \mathrm{~W}$ \\
$\mathbf{R}_{2}$ & $100 \mathrm{k} \Omega, 225 \mathrm{~W}$ \\
$\mathbf{R}_{3}$ & $250 \mathrm{M} \Omega, 5 \mathrm{~W}$ \\
$\mathbf{R}_{4}$ & $2.38 \mathrm{k} \Omega, 1 / 2 \mathrm{~W}$ \\
$\mathbf{R}_{6}$ & $2.0 \mathrm{k} \Omega, 25 \mathrm{~W}$ \\
$\mathrm{C}$ & $4 \times 7.5 \mu \mathrm{F}, 12 \mathrm{kV}$ \\
$\mathrm{L}$ & $5 \mu \mathrm{H}$ or $1200 \mu \mathrm{H}$, air core \\
$\mathrm{D}$ & $20 \mathrm{kV}, 350 \mathrm{~mA}$ diode stack \\
$\mathrm{T}$ & $12 \mathrm{kV}, 60 \mathrm{~mA}$ transformer \\
$\mathrm{S}_{1-3}$ & Gravity operated relay \\
$\mathrm{I}$ & Pearson model 1025 current transformer \\
$\mathbf{F}$ & Thin film in discharge chamber \\
$\mathrm{V}$ & Digital voltmeter \\
\hline
\end{tabular}

conducted at atmospheric pressure in a flowing stream $\left(5 \mathrm{l} \mathrm{min}{ }^{-1}\right)$ of a $40 \% \mathrm{O}_{2}-60 \%$ Ar gas mixture. The cassettes used to support the thin films and their substrates and to provide electrical contact to the metal surface also have been described for both the linear $[1,2]$ and the concentric $[6]$ electrode geometries.

For the concentric geometry, the center electrode, which is not in electrical contact with the metal film, consists of a $0.62-\mathrm{cm}$ diameter high-purity pointed graphite rod. This electrode is replaced daily. The 3-mm thick, ring-shaped outer electrode is also machined from high-purity graphite. The electrode is pressed onto the surface of the $\mathrm{Ag}$ film. Outer electrodes with i.d. ranging from 12.7 to $38 \mathrm{~mm}$ were evaluated. The diameter of the $\mathrm{Ag}$ film is about $40 \mathrm{~mm}$.

\subsection{Optical and electrical monitoring}

A 1.0-m Czerny-Turner spectrometer with a first-order reciprocal linear dispersion of $0.81 \mathrm{~nm} \mathrm{~mm}^{-1}$ was used for all measurements. The discharge chamber was positioned about $67 \mathrm{~cm}$ from the spectrometer entrance slit and no ancillary optics were employed. No spatial resolution was used, and the spectrometer viewed the entire plasma volume. Radiation was detected with a 1P28B photomultiplier tube biased at about $700 \mathrm{~V}$. All waveforms were displayed on a Nicolet Model 2090 digital storage oscilloscope. The gated analog integrator described in [9] was used for time-gated intensity integration.

\subsection{Experimental procedures}

Silver thin films were prepared using polycarbonate filters and polyethylene strips as substrates. Procedures for the vacuum deposition of $\mathbf{A g}$ on these substrates are discussed in [6] and [1], respectively. Properties of the substrates and thin films as well as the discharge conditions used for most of these studies are given in Table 2. 
Table 2. Thin film/substrate properties and discharge conditions

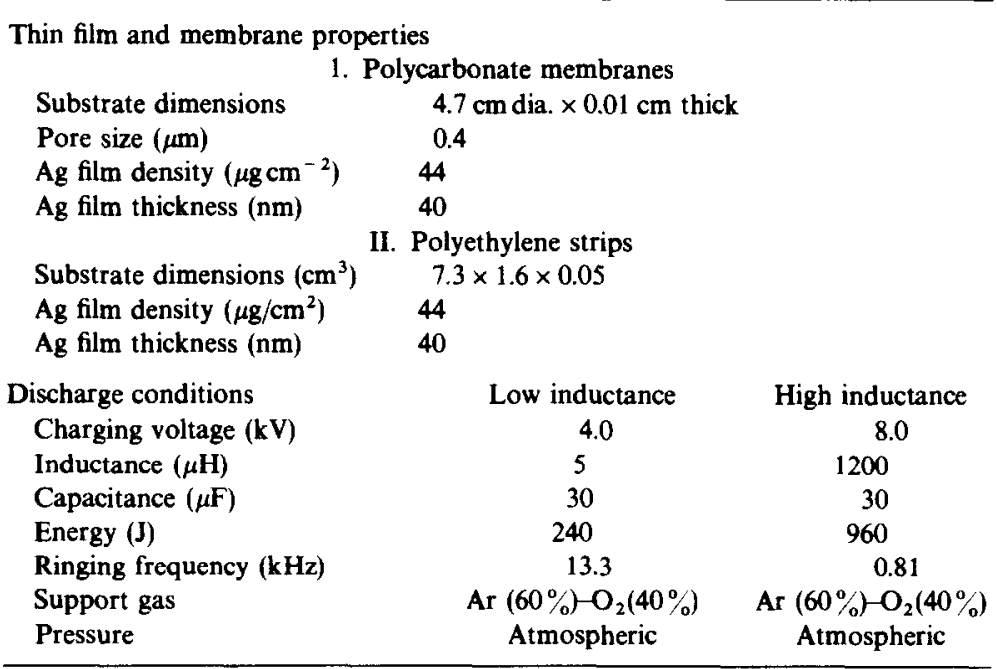

Powder test materials were sorted according to particle size by sieving with micro-mesh sieves in an ATM model L3P sonic sifter. Sample particles were deposited on the thin films using two different procedures. For studies comparing the linear and concentric electrode geometries, 10- $\mu \mathrm{l}$ aliquots of isopropyl alcohol suspensions of the test powders were micropipetted onto the centers of the films. The suspensions were continually stirred with a vortex mixer. Most of the sample was contained in a circle about $1 \mathrm{~cm}$ in diameter. The solvent was evaporated under a heat lamp.

For analytically oriented studies of the concentric electrode system, a more-or-less uniform deposit of the sample over the thin film surface was used to simulate sample particle collection from a fluid medium. The metallized membrane filter was supported in a commercial filter holder with a $4.1-\mathrm{cm}$ diameter chimney. A $10-\mathrm{ml}$ aliquot of stirred powder suspension was poured into the chimney. A slight vacuum then was applied with a water aspirator until the liquid passed through the filter. The filters were then placed in a dessicator where they dried in about 2 min without heating.

\subsection{Materials and reagents}

Silver films were prepared from 25 -mesh $\mathrm{Ag}$ needles with minimum purity of $99.999 \%$. Electrodes were machined from spectroscopic-grade graphite rods. All test materials and calibration standards were reagent grade. The isopropyl alcohol used for sample powder suspensions also was reagent grade.

\section{Comparison of the Linear and Concentric Electrode Geometries}

\subsection{General features}

The very thin and fragile polycarbonate membrane filters, which serve as substrates for the thin metal films, are destroyed during the discharge, but no impurity radiation is observed from them [3]. This suggests that the membrane is physically disintegrated by the plasma shock waves but is not heavily sampled by the plasma. The more robust polyethylene substrates used with the linear thin films show only slight discoloration from the discharge. However, the surface probably is etched to some extent by the plasma.

Figure 2 shows sectioned views of the linear (a) and concentric (b) electrode geometries. They are not drawn to scale so that certain features can be seen more clearly. In the linear geometry, the graphite electrodes are positioned at opposite ends of the rectangular film. The film is supported on a polyethylene substrate on which it is initially vaccum deposited. Plastic shields are used to prevent sparkover to metallic parts of the cassette which are not shown in the figure. The plasma current channel is anchored to the electrodes and passes through the narrow channels between the bottom of the shields and the substrate surface. The plasma can expand freely in the region between the shields. Radiation is observed normal to the plane of the figure, and for the studies reported here, the spectometer viewed the entire plasma volume. The initial polarity of the electrodes has no effect with this configuration. 
(a)

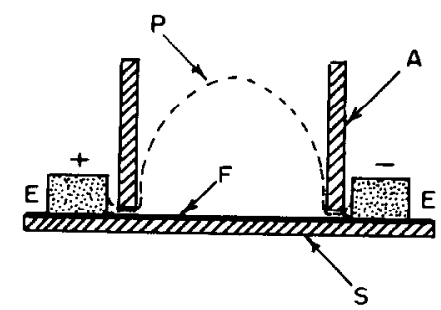

(b)

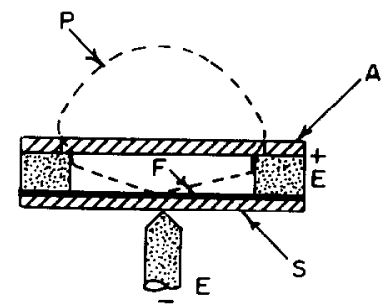

Fig. 2. Sectioned views of the linear (a) and the concentric (b) electrode geometries. (F) thin film; (S) substrate; (E) electrode; (A) plastic insulation to prevent sparkover; (P) plasma.

With the concentric electric geometry, a radial current path is established in the thin film prior to vaporization and in the plasma after vaporization. This results in much greater current density near the center electrode. With the linear geometry, the current density does not vary significantly in the thin film or in the plasma along the electric field axis. Previous studies with the linear geometry [8] showed a large voltage spike across the electrodes at the time of film vaporization. This appears to be an inductive effect caused by the rapid conversion of the film from a conducting solid or liquid to a gaseous dielectric. The voltage then quickly falls when breakdown occurs through the metal vapor. This voltage spike is nearly absent with the concentric geometry. This is probably the result of film vaporization proceeding gradually from the region near the center electrode where resistive heating should be greatest to the region near the ring electrode. Thus, an arc probably is formed from the center electrode to the edge of the unvaporized film. The arc expands as the film continues to vaporize until it reaches the outer electrode.

Relatively good shot-to-shot reproducibility of both electrical waveforms and continuum background intensity with the concentric electrode system suggests that the plasma current is more-or-less uniform at the outer electrode rather than forming arc channels to a few spots on the ring electrode.

The lower portion of the plasma from the concentric electrode arrangement is masked by the 3.0-mm thick ring-shaped outer electrode and by the $0.5-\mathrm{mm}$ thick plastic ring cemented to the top of the electrode. Thus this region is not observed by the spectrometer. Much of the current channel probably is in the masked region. The outer electrode also confines the lower portion of the plasma, and the plasma appears to emanate from the graphite ring.

\subsection{Comparison of discharge current and radiation waveforms}

Discharge current waveforms and the time variation of radiation at selected wavelengths were obtained for both electrode geometries and for both the low-inductance and the highinductance discharge circuits. Figure 3 shows the current waveforms and the continuum background radiation at about $403 \mathrm{~nm}$ for both geometries using low-inductance discharges. The current waveforms are significantly different. The discharge current for the concentric geometry (d) rings considerably longer than for the linear geometry (b), and its peak value in the first half cycle is about $25 \%$ greater. The ringing frequency for the linear geometry is about $12.7 \mathrm{kHz}$ and about $13.3 \mathrm{kHz}$ for the circular geometry.

These current waveform differences suggest that the plasma impedance is lower with the concentric-electrode geometry. This may be due to the greater area of electrode exposed to the plasma and the attendant reduction in the space-charge impedance.

The peak continuum intensity during the first current half cycle is about a factor of six greater for the linear geometry. The difference becomes much smaller, however, after the 
(a)

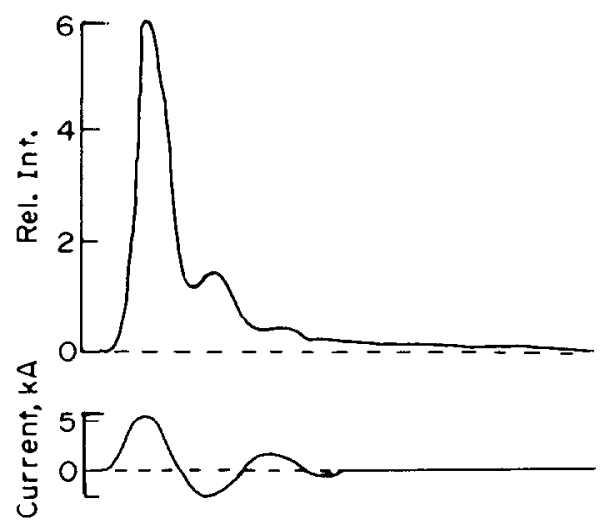

(c)

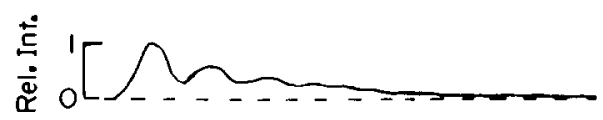

(d)

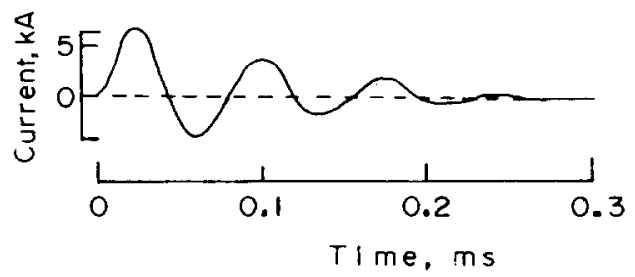

Fig. 3. Continuum background and discharge current waveforms for low-inductance discharges.

(a) continuum background radiation for linear geometry; (b) current for linear geometry;

(c) continuum background radiation for concentric geometry; (d) current for concentric geometry.

second half cycle. A long, low-intensity tail is observed after cessation of the discharge current for the linear-geometry plasma. The much lower continuum intensity observed early in time with the concentric-electrode geometry probably is a result of the masking by the ring electrode. Assuming a plasma expansion velocity of about $100 \mathrm{~m} \mathrm{~s}^{-1}$ for the discharge conditions used here [10], about $30 \mu \mathrm{s}$ would be required for the plasma to move beyond the 3-mm thick ring electrode. Since the plasma current density is greatest during the first current half cycle, the continuum intensity also is a maximum during this time, and it is this very intense continuum that is most effectively masked by the ring electrode.

The effects of plasma impedance on the peak current and frequency of the current waveform are significant when the circuit inductance is low. These effects are greatly reduced when $1200 \mu \mathrm{H}$ of inductance are added to the circuit. This is illustrated in Fig. 4 where waveforms analogous to those in Fig. 3 are presented for high-inductance discharges. Here, the peak current and frequency differ by only a few percent. The relative difference in discharge duration, however, is about the same as for the low-inductance discharges. This is expected since the damping constant for an underdamped RCL tank circuit is proportional to resistance [11]. Note that the duration of the high-inductance discharges typically is about 25 -fold greater than for the low-inductance discharges. Thus, significant differences in plasma properties are not surprising.

Differences in the continuum background radiation are significant for the two geometries with the high-inductance discharges but not as large as the differences with the lowinductance discharges. Both the peak continuum intensity and the value integrated over the duration of the discharge are about a factor of two greater for the linear electrode geometry. Again, in part, this may be due to masking of the lower portion of the plasma by the ringshaped electrode. 
(a)

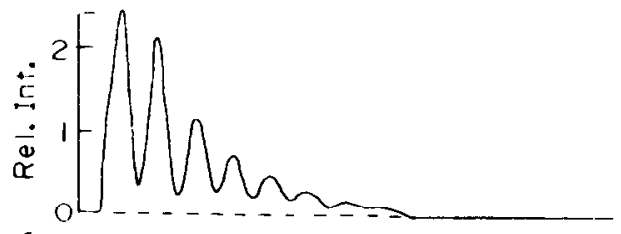

(b)

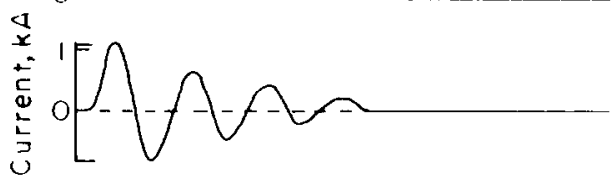

(c)

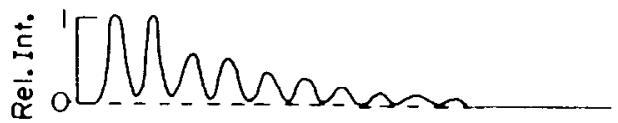

(d)

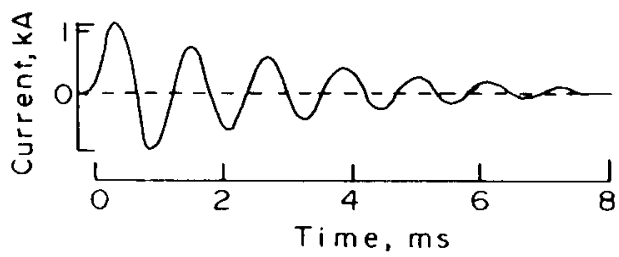

Fig. 4. Continuum background and discharge current waveforms for high-inductance discharges.

(a) continuum background radiation for linear geometry; (b) current for linear geometry;

(c) continuum background radiation for concentric geometry; (d) current for concentric geometry.

\subsection{Ion and neutral atom analyte radiation comparison}

Analyte neutral atom and ion line radiation was investigated for both electrode geometries and for both values of circuit inductance. A $1.0-\mu \mathrm{g} \mathrm{Mn}$ powder sample was applied to the center of each film as a suspension of particles which passed through a 5- $\mu$ m pore diameter sieve. In each case, the results from four experiments were corrected for background and averaged. Figure 5 shows temporal radiation profiles for the Mn 344.2-nm ion line (a and c) and the 403.1-nm neutral atom line (b and d) using low-inductance discharge conditions. A current waveform from the concentric electrode system (e) also is provided as a time reference.

With both electrode geometries, the analyte line radiation is detectable for more than $0.2 \mathrm{~ms}$ after current flow has ceased, and the peak line radiation occurs after the peak in the continuum background radiation. While the oscillations in the $\mathrm{Mn}$ ion line radiation are more significant with the concentric-electrode geometry, they are not a dominant feature in any of the analyte line radiation profiles with the low-inductance discharges.

Perhaps the most interesting feature in Fig. 5 is the rather intense Mn neutral atom line radiation late in the discharge with the concentric-electrode geometry. The upper excited state responsible for this line appears to be pumped during the first two or three current half cycles. The intensity then remains fairly constant until the end of the discharge and then decreases rather slowly for several tenths of a millisecond. SCHEELINE et al. [12] have observed ground-state metal ions for significant times after the end of current flow in a high-voltage spark discharge to a metal electrode. This may also explain the high-intensity neutral atom afterglow observed in Fig. 5. If diffusion late in the discharge is not ambipolar, these ions may be metastable with respect to ion-electron recombination and serve as a long-term source for the population of neutral atom states. Similar radiation patterns have been observed for neutral atom lines from other elements.

The situation is quite different with the high-inductance discharges as shown in Fig. 6. Here, for both the linear and the concentric geometries, neutral atom and ion radiation fall below detectability at about the time of current cessation. This is not surprising, however, if one considers the much greater duration and more gradual change in discharge current for 
(a)

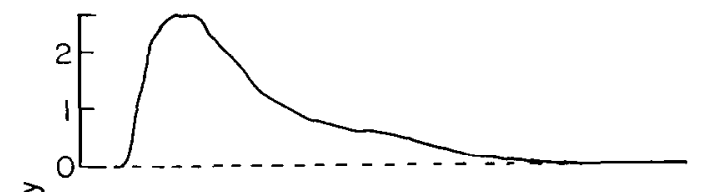

(b)

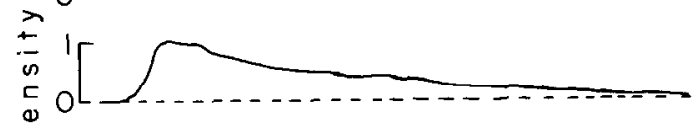

(c)

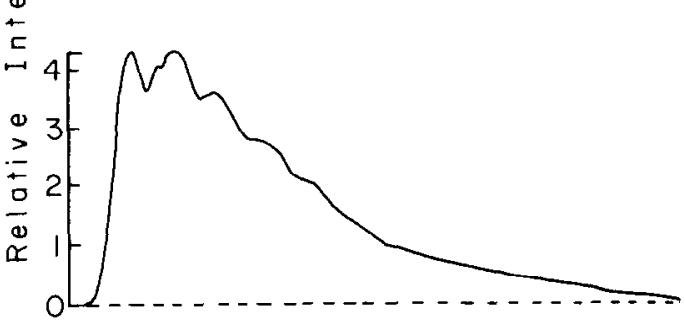

(d)

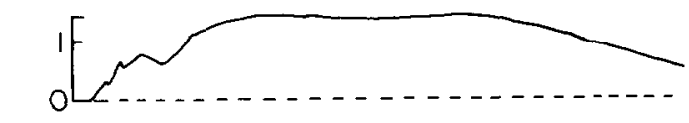

(e)

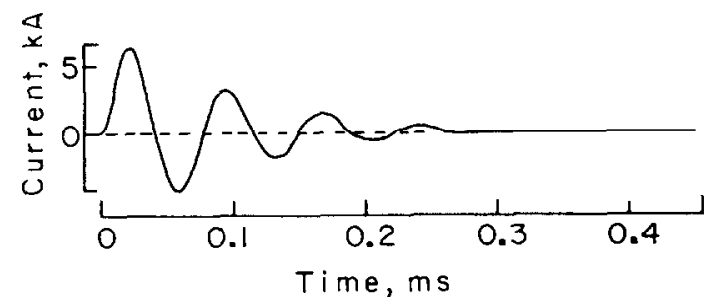

Fig. 5. Line radiation waveforms for low-inductance discharges. (a) Mn II $344.2 \mathrm{~nm}$ for linear geometry; (b) Mn I $403.1 \mathrm{~nm}$ for linear geometry; (c) Mn II $344.2 \mathrm{~nm}$ for concentric geometry;

(d) $\mathrm{Mn}$ (I) $403.1 \mathrm{~nm}$ for concentric geometry; (e) discharge current for concentric geometry.

(a)

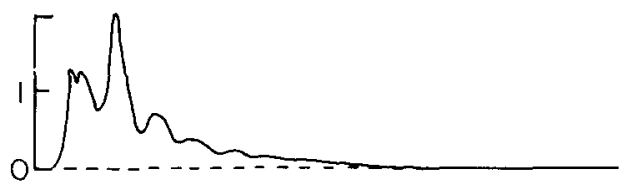

(b)
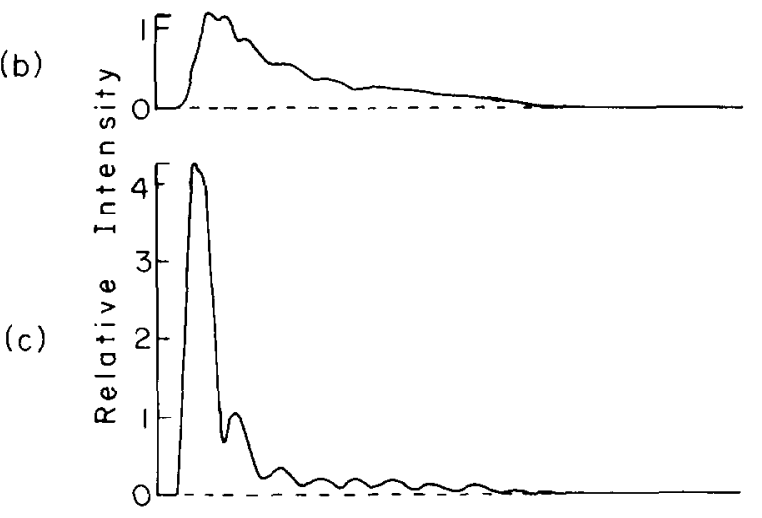

(d)

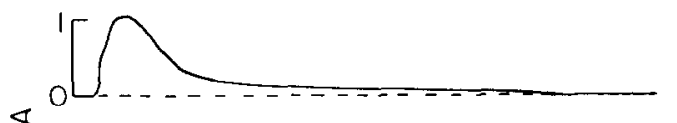

(e)

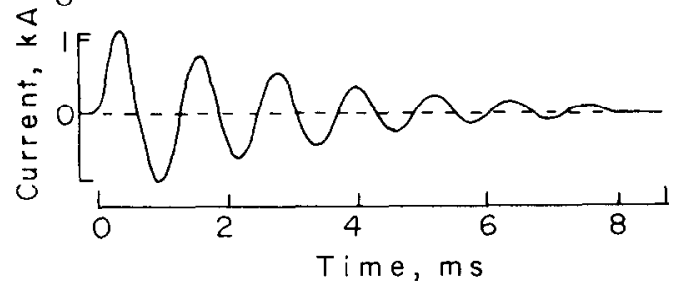

Fig. 6. Line radiation waveforms for high-inductance discharges. (a) $\mathrm{Mn}$ II $344.2 \mathrm{~nm}$ for linear geometry; (b) Mn I $403.1 \mathrm{~nm}$ for linear geometry; (c) Mn II $344.2 \mathrm{~nm}$ for concentric geometry; (d) Mn I $403.1 \mathrm{~nm}$ for concentric geometry; (e) discharge current for concentric geometry. 
the high-inductance discharge conditions. Oscillations in the $\mathrm{Mn}$ ion line intensity are more pronounced with the high-inductance discharge. Again, this is probably the result of the slower changes in discharge current with respect to energy partitioning processes. Finally, both neutral atom and ion line intensities are relatively greater early in the discharge with the concentric-electrode geometry. This is particularly apparent for the ion line. This could be the result of either more rapid sample atomization early in the discharge or more rapid vapor loss late in the discharge with the concentric geometry.

\section{Concentric Geometry Parametric Studies}

The variations of line intensity and line-to-background intensity ratios with respect to initial capacitor voltage, discharge energy, circuit inductance, plasma support gas and pressure have been described for the linear geometry system [13]. Results with the concentric-electrode geometry are not significantly different and will not be discussed here. The initial electrode polarity and the inside diameter of the ring-shaped outer electrode are potentially important parameters unique to the concentric system.

\subsection{Initial electrode polarity}

The concentric-electrode geometry allows the setting of the intitial polarity of the two electrodes. While this is also possible with the linear geometry, no spectral differences are observed because of the symmetry involved. Figure 7 shows the effect of initial electrode polarity for the concentric-electrode system on the ion (a) and neutral atom (b) line radiation from a 100-ng sample of $\mathrm{Mn}$ powder which passed through a 5- $\mu \mathrm{m}$ pore sieve. Lowinductance discharges were used, and the continuum background waveform (c) is provided as a reference. The traces labeled " $A$ " were obtained with the center electrode initially anodic, and those labeled " $\mathrm{C}$ " had the center electrode initially cathodic. The intensity is consistently greater with the center electrode initially cathodic, and when integrated over the duration of the discharge, 21 and $25 \%$ increases are observed for the neutral-atom and ion line, respectively.

Figure 8 shows similar plots for the high-inductance discharges. Again, time-integrated intensity values are significantly greater when the center electrode is initially cathodic, but the instantaneous intensity values change with each current half cycle so that greater intensity is observed when the center electrode is cathodic during a given half cyclc.

The general trend toward greater line radiation intensity when the center electrode is initially cathodic is not surprising. Recall that the powder sample was deposited as a relatively small spot directly over the center electrode. Because of its small size, the plasma current density may be very large near the center electrode. Cathode-layer enrichment often is observed with direct-current arc excitation if a small sample is vaporized from a cathodic graphite electrode $[14,15]$. This is probably the result of attraction of positive analyte ions to the negative region of the cathode space charge. Sampling of a metallic electrode by a highvoltage capacitive spark has been shown to be more efficient when the electrode is cathodic $[16,17]$. The reason for this is not completely clear but may be the result of positive-ion bombardment of the cathode surface.

The difference in behavior of the low-inductance and high-inductance discharges with respect to initial electrode polarity may be the result of the large differences in ringing frequency. The relatively low frequency of the high-inductance discharge may allow sufficient time during each half cycle for the migration of analyte ions to the space charge region near the cathodic electrode. Since the continuum background intensity is independent of initial electrode polarity, the center electrode was made initially cathodic for all further analytical studies.

[13] R. D. SACKS and D. V. DuChane, Anal. Chem. 50, 1757 (1978).

[14] R. Avni and A. Boukobza, Appl. Spectrosc. 23, 483 (1969).

[15] W. C. Myers and W. M. HenRY, Ultrapurification of Semiconductor Materials, p. 349. Macmillan, New York (1962).

[16] J. P. Walters, Appl. Spectrosc. 23, 317 (1969).

[17] T. TAKahashi and K. Yoshino, Trans. Natn. Res. Inst. Metals 10, 53 (1968). 
(a)

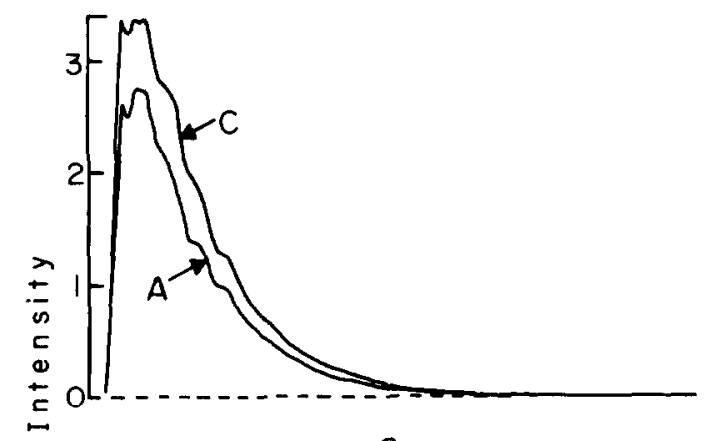

(b)

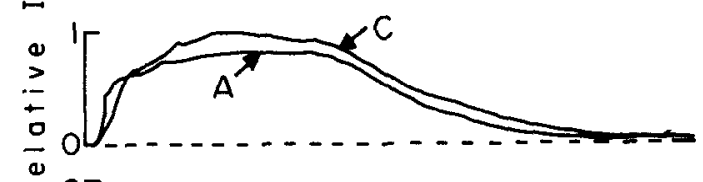

(c)

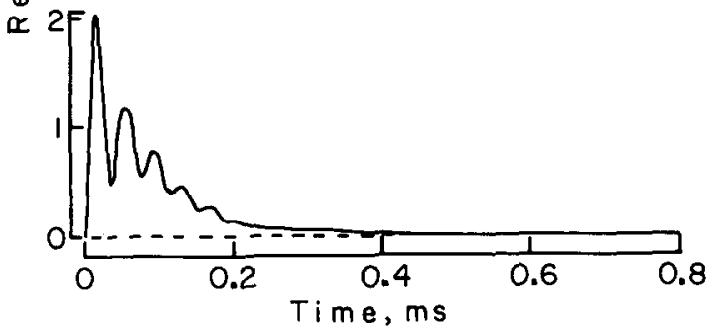

Fig. 7. Electrode polarity effects for low-inductance discharges. (a) Mn II $344.2 \mathrm{~nm}$ line; (b) Mn I $403.1 \mathrm{~nm}$ line; (c) continuum background. For traces marked $\mathrm{C}$, the center electrode was initially cathodic; while for those marked $A$, it was initially anodic.

(a)

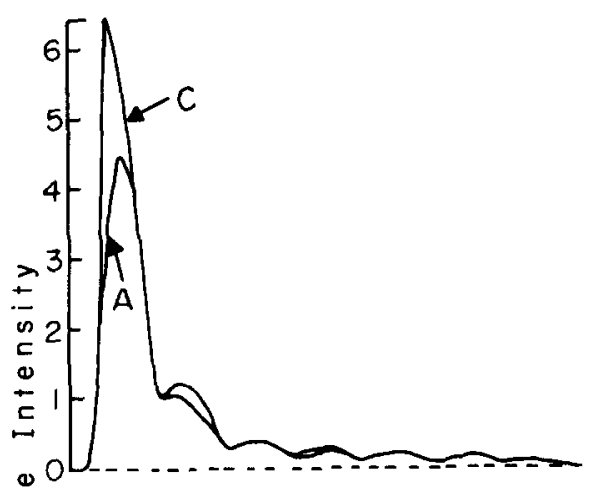

(b)

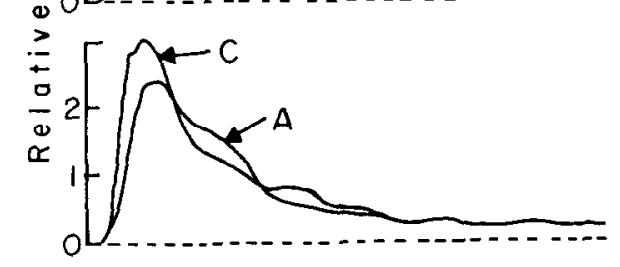

(c)

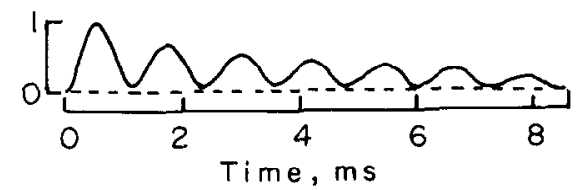

Fig. 8. Electrode polarity effects for high-inductance discharges. (a) $\mathrm{Mn}$ II $344.2 \mathrm{~nm}$ line; (b) $\mathrm{Mn} \mathrm{I}$ 403.1 nm line; (c) continuum background. For traces marked $C$, the center electrode was initially cathodic; while for those marked $\mathrm{A}$, it was initially anodic. 


\subsection{Electrode size effects}

With the concentric-electrode arrangement, dimensions of the plasma can be controlled in part by the diameter of the outer electrode. Recall that the plasma current path is primarily radial and that current density is probably lower near a larger-diameter outer electrode than near a smaller-diameter one. Five outer-electrode inside diameters were tested ranging from 12.7 to about $38 \mathrm{~mm}$. Since the $\mathrm{Ag}$ film is about $40 \mathrm{~mm}$ in diameter, from 10 to about $90 \%$ of the film is exposed to the plasma for this range of outer electrode diameters.

Relative intensity vs exposed film area data for high-inductance discharges are plotted in Fig. 9. Each point represents an average from four experiments. Continuum background intensity is plotted for two integration intervals, $0-4.3 \mathrm{~ms}$ (plot 1) and 1.2-4.3 ms (plot 2). The intensity of the V 311.8-nm ion line from 2- $\mu \mathrm{g}$ VC powder samples placed near the center of the film also is plotted (plot 3). Intensity data for this plot are integrated from 0 to $4.3 \mathrm{~ms}$. With a centrally applied sample, the sample is located near the center electrode. Current density at this electrode should be nearly independent of the outer electrode diameter. Note that over a nearly 9-fold range of thin film area and mass, there is only about a $35 \%$ increase in intensity with increasing area. Since excited state populations are very sensitive to the electron temperature of the plasma, these data suggest that electron temperature in analytically useful regions near the center electrode is not strongly dependent on the dimensions of the thin film.

If integration of the continuum background is started at the start of the discharge, there is about a $70 \%$ increase in intensity with increasing area over the area range investigated. This increase is surprisingly modest in light of the 9-fold increase in the mass of $\mathrm{Ag}$ vaporized by the plasma. Silver vapor should be a principal plasma component for all the studies reported here. When integration is delayed until the start of the third current half cycle, the increase in continuum intensity with electrode size is only half as large as in the former case. This is probably because the plasma has expanded considerably, and most of its volume may no longer be strongly influenced by the electrode space charge. Note that line-to-background ratios are relatively independent of film area for either integration interval.

\section{Analytical Considerations}

\subsection{Sample introduction and standardization}

Since solutions pass directly through the membrane filter, only particle samples which are retained on the filter surface can be determined by this technique. This applies to calibration

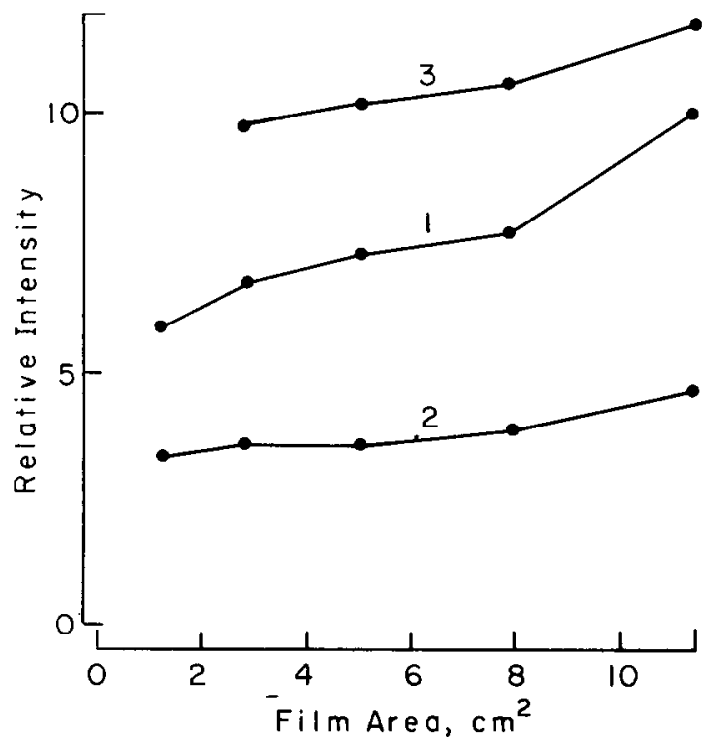

Fig. 9. Electrode size effects for high-inductance discharges. (1) continuum intensity for time interval $0-4.3 \mathrm{~ms}$; (2) continuum intensity for time interval $1.2-4.3 \mathrm{~ms}$; (3) VII $311.8 \mathrm{~nm}$ line for time interval $0-4.3 \mathrm{~ms}$. 
standards as well as to samples. Since compound form effects and concomitant effects are minimal in electrically vaporized thin film plasmas $[1,3]$, any reagent or other material containing the element of interest can be used as a calibration standard as long as the chemical form of the element is insoluble in the medium used to prepare the powder suspensions. Pure metals, which often are commercially available as fine powders, have worked well in our laboratory. SWAN and SACKs [6] used $\mathrm{Pb}$ powder standards with the concentric-electrode system for the determination of $\mathrm{Pb}$ in an urban airborne particulate sample. Using delayed intensity integration, recoveries of 99 and $109 \%$ were obtained using the low-inductance and high-inductance discharges, respectively.

The introduction of powder standards as suspensions that are filtered through the metallized membrane is very convenient and produces a powder distribution on the thin film surface which is similar to that obtained from samples collected by filtration from a fluid medium. However, statistical problems become important for low-mass standards. A 10-ng standard of a typical inorganic material with a density of about $5 \mathrm{~g} \mathrm{~cm}^{-3}$ would contain only about $305-\mu \mathrm{m}$ diameter particles. The statistical variation in the number of particles from a randomly drawn aliquot of a stirred suspension would be too large for some applications. This clearly points out the need for standards of very small particle size, particularly when low mass standards are involved.

\subsection{Particle size and sample size effects}

GolDBERG and SACKS [1] have shown that particle size effects can be virtually eliminated for the linear-electrode geometry by adding a large inductance to the discharge circuit. This reduces the damping constant of the $\mathrm{RCL}$ tank and thus increases the plasma/sample interaction time. After about $2 \mathrm{~ms}$, sample particles as large as about $30 \mu \mathrm{m}$ are vaporized completely and produce the same intensity as smaller particles and aqueous solution residues of equal analyte mass. Thus delayed intensity integration is very effective in eliminating particle size effects. The effects of large sample loadings also are reduced in this way, and 1-mg samples show minimal concomitant effects when intensity integration is delayed about $2 \mathrm{~ms}$ using a $1200-\mu \mathrm{H}$ discharge.

Recently, Swan and SACKS [6] showed that high circuit inductance and delayed integration also are effective in reducing sample loading effects for electrically-vaporized thin-film plasmas using the concentric-electrode geometry. However, these experiments were conducted with small-particle samples and no specific particle size effect studies were reported.

Figure 10 shows radiation profiles for the $\mathrm{Mn} 344.2-\mathrm{nm}$ ion line from 2- $\mu \mathrm{g} \mathrm{Mn}$ powder samples of various particle sizes. These profiles were obtained using low-inductance discharges with the concentric electrode geometry. Note that particle size effects are significant and that the radiation profiles do not change much relative to each other after the second current half cycle. Thus delayed integration would not be useful in reducing these effects.

Table 3 gives the intensity values for the various particle sizes integrated over the duration of the discharge for both the Mn profiles shown in Fig. 10 and for the V 311.1-nm ion line from $2-\mu \mathrm{g}$ VC samples. Intensity values are normalized to 100 for the smallest particles. The greater than expected intensity from the largest VC particles is the result of the particle structure. Electron microscopy of VC particles [3] has shown that particles in the 20-30 $\mu \mathrm{m}$ size range are really aggregates of smaller particles which presumably have greater surface area and thus vaporize more rapidly than the $15-20 \mu \mathrm{m}$ particles. With the exception, the more refractory nature of VC is reflected in a more rapid loss in intensity with increasing particle size than the relatively low-boiling Mn metal.

The differences in analyte intensity between the smallest and the largest particles as indicated in Table 3 are not as extreme as reported for the linear geometry under similar discharge conditions, where corresponding intensity values spanned over a decade of values [1].

Figure 11 shows radiation profiles for the same $\mathrm{Mn}$ ion line for three particle sizes but using high-inductance discharges with the concentric-electrode geometry. Note that the greatest emission intensity from the $<5-\mu \mathrm{m}$ particlcs (a) occurs during the first current half 

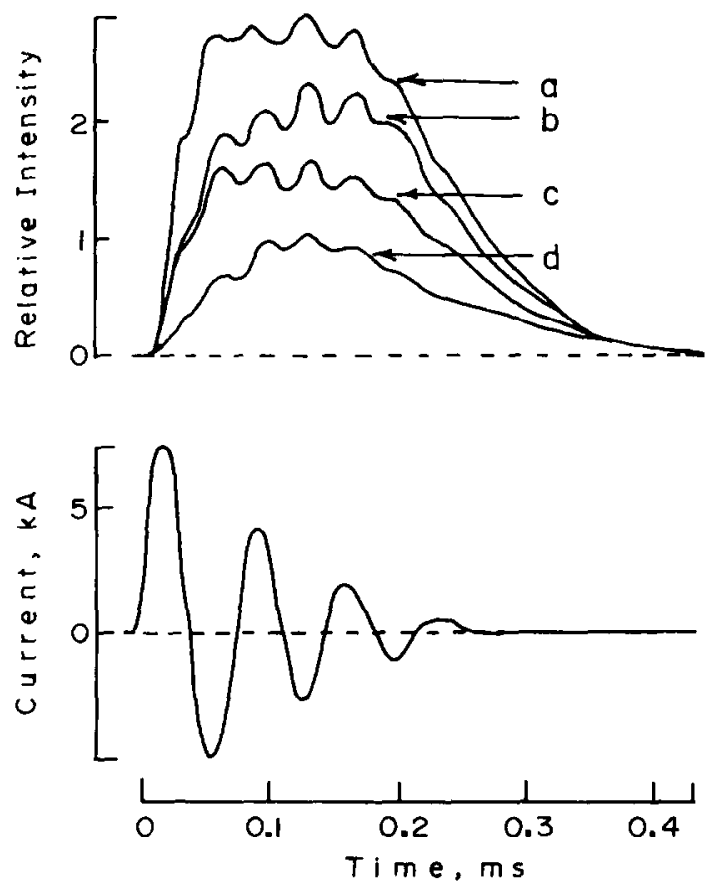

Fig. 10. Radiation profiles for the Mn II 344.2-nm line for 2- $\mu \mathrm{g}$ Mn powder samples using lowinductance discharges. (a) 5-10 $\mu \mathrm{m}$ particles; (b) 10-15 $\mu \mathrm{m}$ particles; (c) 15-20 $\mu \mathrm{m}$ particles; (d) 20-30 $\mu \mathrm{m}$ particles.

Table 3. Particle size effects for low-inductance discharges with the concentric-electrode geometry

\begin{tabular}{|c|c|c|}
\hline \multirow{2}{*}{$\begin{array}{l}\text { Particle size range } \\
\qquad(\mu \mathrm{m})\end{array}$} & \multicolumn{2}{|c|}{ Relative intensity } \\
\hline & VC & $\mathrm{Mn}$ \\
\hline$<5$ & 100 & 100 \\
\hline $5-10$ & 97 & 100 \\
\hline $10-15$ & 69 & 76 \\
\hline $15-20$ & 41 & 57 \\
\hline $20-30$ & 56 & 31 \\
\hline
\end{tabular}

cycle. As the sample particles get larger, the time of peak intensity is progressively delayed. This was also observed for high-inductance discharges with the linear electrode geometry [1]. What was not observed, however, is the greater intensity late in time from the larger particle samples seen in Fig. 11. With the linear geometry, the emission profiles for the different particle sizes became indistinguishable after about three current half cycles $(1.8 \mathrm{~ms})$.

Table 4 lists relative intensities of the $V 311.1-\mathrm{nm}$ and $\mathrm{Mn} 344.2-\mathrm{nm}$ lines from 2- $\mu \mathrm{g}$ samples of various particle sizes of $\mathrm{VC}$ and $\mathrm{Mn}$ using several integration time intervals. Highinductance discharges and the concentric-electrode geometry were used in all cases. All values are individually normalized for each integration interval to give a value of 100 for the smallest particles. Note that the range of values with respect to particle size becomes progressively larger with increasing delay in the integration interval, and the most favorable results (minimum particle size effects) occurs with integration over the entire discharge. Apparently the greater intensity late in time from the larger particles is nearly offset by lower intensity during the first half cycle.

While the particle size effects observed here under the most favourable conditions with the high-inductance discharges are somewhat larger than values reported using delayed integration with the linear electrode geometry, they are not extreme considering the fairly 
(a)

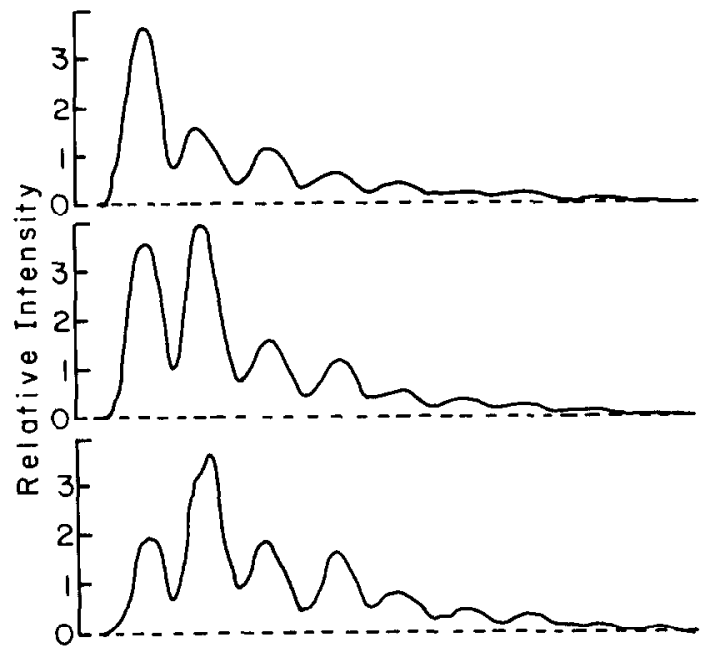

(d)

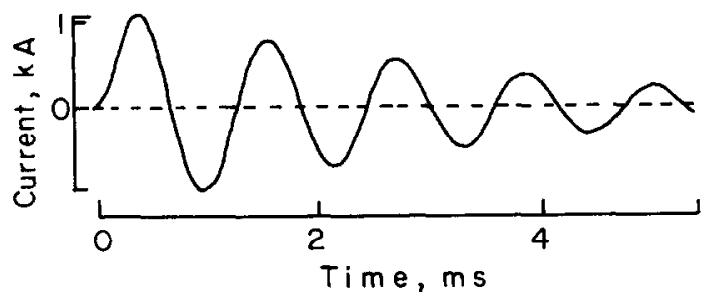

Fig. 11. Radiation profiles for the Mn II 344.2-nm line for 2- $\mu \mathrm{g}$ Mn powder samples using highinductance discharges. (a) $<5 \mu \mathrm{m}$ particles; (b) 10-15 $\mu \mathrm{m}$ particles; (c) 20-30 $\mu \mathrm{m}$ particles; (d) current waveform.

Table 4. Particle size effects for high-inductance discharges with the concentricelectrode geometry

\begin{tabular}{ccccc}
\hline $\begin{array}{c}\text { Particle size range } \\
(\mu \mathrm{m})\end{array}$ & $0-4.3 \mathrm{~ms}$ & $0.6-4.3 \mathrm{~ms}$ & $1.2-4.3 \mathrm{~ms}$ & $1.8-4.3 \mathrm{~ms}$ \\
\hline & \multicolumn{4}{c}{ Vanadium carbide, V II 311.1 $\mathrm{nm}$} \\
$<5$ & 100 & 100 & 100 & 100 \\
$5-10$ & 144 & 172 & 177 & 169 \\
$10-15$ & 120 & 191 & 229 & 190 \\
$15-20$ & 123 & 223 & 226 & 264 \\
$20-30$ & 106 & 213 & 288 & 292 \\
& & Manganese, Mn II 344.2 $\mathrm{nm}$ & \\
$<5$ & 100 & 100 & 100 & 100 \\
$5-10$ & 138 & 138 & 122 & 146 \\
$10-15$ & 154 & 181 & 161 & 191 \\
$15-20$ & 170 & 238 & 243 & 262 \\
$20-30$ & 153 & 233 & 237 & 332 \\
\hline
\end{tabular}

wide particle size range considered in this study. Note that a $30-\mu \mathrm{m}$ diameter spherical particle has 36 times the surface area and 216 times the mass of a $5 \mu \mathrm{m}$ particle.

\subsection{Analytical curves}

Analytical curves (log-log plots) were prepared for several elements using small-particle $(<5 \mu \mathrm{m})$ powder standards which were deposited uniformly over the entire thin film surface. A 38-mm i.d. outer electrode was used for these studies. At each concentration, intensity values were averaged for four experiments. Both high-inductance and low-inductance 
discharges were evaluated. Reasonably good correlations were obtained with correlation coefficients typically in the $0.97-0.99$ range for a four-point or a five-point curve. Ion lines usually produce slopes in the range $0.85-1.10$, but neutral atom lines often result in significantly lower slopes. This may be the result of self absorption in the large, rather homogeneous thin film plasma.

Figure 12a shows typical analytical curves for $\mathrm{Pb}$ and $\mathrm{V}$ using high-inductance discharge conditions. Vanadium curves from VC powder standards were obtained for the $311.1-\mathrm{nm}$ ion line using two different integration intervals (1.2-4.3 ms for curve 1 and $0-4.3 \mathrm{~ms}$ for curve 2$)$. The former interval begins at the start of the third current half cycle of the discharge. The curves are similar but the log-log slope is slightly larger with the delayed integration. Curves 3 and 4 were obtained from the $\mathrm{Pb} 220.4 \mathrm{~nm}$ ion line from $\mathrm{Pb}$ powder standards. The integration intervals were $0-4.3 \mathrm{~ms}$ for curve 3 and $1.2-4.3 \mathrm{~ms}$ for curve 4 . Integration from the start of the discharge results in a slope of 0.88 ; while with delayed integration, the slope is 1.04 .

Figure $12 \mathrm{~b}$ shows $\mathrm{Pb}$ analytical curves for low-inductance discharges. Curve 1 is for the 220.4-nm ion line integrated from 0.125 to $0.50 \mathrm{~ms}$ after the start of the discharge. This integration interval begins in the third half cycle of the discharge. Curve 2 is for the same line but integrated over the entire duration of the discharge. Curve 3 is for the 283.3-nm neutralatom line integrated over the entire discharge. Curves 1 and 2 are very similar with slopes of 0.97 and 0.93 , respectively. The neutral-atom line has a slope of only 0.67 .

(b)
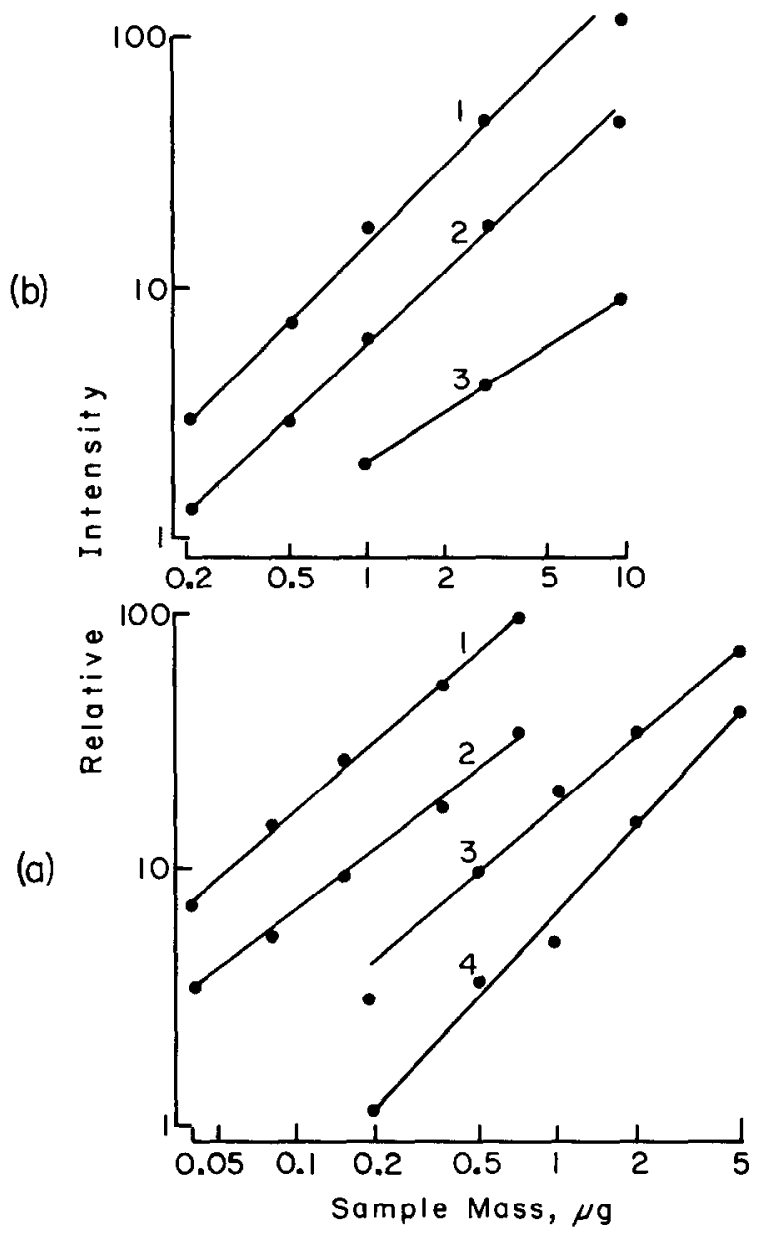

Fig. 12. Analytical curves using (a) high-inductance discharges and (b) low-inductance discharges. (a-1) V II 311.1-nm line integrated from 1.2 to $4.3 \mathrm{~ms}$; (a-2) V II 311.1-nm line integrated from 0 to $4.3 \mathrm{~ms}$; (a-3) $\mathrm{Pb}$ II 220.4-nm line integrated from 0 to $4.3 \mathrm{~ms}$; (a-4) $\mathrm{Pb}$ II 220.4-nm line integrated from 1.2 to $4.3 \mathrm{~ms}$; (b-1) Pb $1 \mathrm{2} 220.4-\mathrm{nm}$ line integrated from 0.125 to $0.50 \mathrm{~ms}$; (b-2) Pb II integrated from 0 to $0.50 \mathrm{~ms}$; (b-3) $\mathrm{Pb}$ I 283.2-nm line integrated from 0 to $0.50 \mathrm{~ms}$. 
As shown in Fig. 5, the low-inductance discharge conditions with the concentric-electrode geometry result in neutral-atom line radiation that persists at relatively high intensity for more than $0.2 \mathrm{~ms}$ after the discharge current has ceased. The line-to-background intensity ratio is quite favorable during this time. Several analytical curves were prepared for lowinductance discharges using integration intervals which begin $0.3 \mathrm{~ms}$ after the start of the discharge. For all lines studied, analytical curves had slopes of much less than unity, and in many cases, the $\log -\log$ plots were nonlinear. In general the slopes are too small to be of analytical utility. Self-absorption is suspected in these cases.

\section{Conclusions}

Both radiative and electrical properties of electrically vaporized thin film plasmas from the concentric-electrode system are significantly different than plasmas from the linear electrode system. Electrode polarity effects and a radial current path with current density dependent on the outer electrode diameter with the concentric-electrode system are largely responsible for the differences. Increased sampling efficiency or positive-ion enrichment may be responsible for the greater analyte intensity observed when the center electrode is initially cathodic.

The variation in current density with position in the plasma may be the reason for the particle-size-effect differences observed for the two electrode geometries. Since most real samples will have a significant range of particle sizes, a powder standard with a particle size distribution similar to that obtained from typical grinding and milling operations seems desirable for use with the concentric geometry. However, SwAN and SACKs [6] were able to obtain $\mathrm{Pb}$ recoveries in the $75-110 \%$ range when $\mathrm{Pb}$ in an urban airborne particulate sample with a broad particle size distribution was determined using a $\mathrm{Pb}$ powder standard with a maximum particle size of $5 \mu \mathrm{m}$. If the mass of the standard is so small that particle sampling statistics dominate the measurement variance, a small particle standard may be preferred even if the sample is composed of a range of particle sizes.

Powers of detection with the metallized membrane substrates typically are a factor of 2-10 poorer than with metal films on solid substrates. This is not because of lower line-tobackground intensity ratios but rather because of larger shot-to-shot relative standard deviations. Values typically are in the $\pm 20-30 \%$ range for plasmas generated from metallized membrane filters and $\pm 5-15 \%$ for plasmas from films on solid substrates. This may be the result of plasma instabilities and/or scattering caused by the mechanical disintegration of the membrane.

The concentric-electrode geometry with metallized membrane filters already has proven useful for the direct determination of $\mathrm{Pb}$ in air-borne particulate material. Other applications to solid particles suspended in fluid media should be straightforward. However, films on solid substrates and the linear electrode geometry are preferred for other solid samples because of greater precision, lower detection limits and greater standardization flexibility.

Acknowledgement - The authors acknowledge support of this study by the National Science Foundation through grant No. CHE 78-25542. 